BULLETIN OF THE

AMERICAN MATHEMATICAL SOCIETY

Volume 78, Number 5, September 1972

\title{
SINGULARITY SUBSCHEMES AND GENERIC PROJECTIONS
}

\author{
BY JOEL ROBERTS ${ }^{1}$
}

Communicated by Michael Artin, April 3, 1972

Let $k$ be an algebraically closed field, and let $\boldsymbol{P}^{n}$ be projective $n$-space over $k$. Let $V^{r} \subset P^{n}$ be a smooth projective variety. It is known that $V$ can be embedded in $\boldsymbol{P}^{2 r+1}$ (cf. [2]), but there are smooth $r$-dimensional varieties which cannot be embedded in $\boldsymbol{P}^{2 r}$.

Let $r \leqq m \leqq \min (2 r, n-1)$, and let $\pi: V \rightarrow \boldsymbol{P}^{m}$ be induced by projection from an $(n-m-1)$-subspace $L \subset P^{m}$ with $L \cap V=\varnothing$. As in [4], we ask what can be said about $\pi$ when $L$ is chosen generically, i.e. $L$ is chosen from some dense open subset of the corresponding Grassmann variety. In the case that $m \geqq r+1$, the problem is to describe the local nature of the singular locus of $V^{\prime}=\pi(V)$. This is of interest because $V^{\prime}$ can be chosen to be birational to $V$. Specifically, we would like to describe the structure of the local rings $\hat{\mathcal{O}}_{V^{\prime}, y}$ for closed points $y \in V^{\prime}$.

For $i>0$, let $S_{i} \subset V$ consist of all points $x$ at which the tangent map has rank $\leqq r-i$. Thus

$$
S_{i}=\left\{x \in V \mid \operatorname{dim}_{k(x)}\left(\Omega_{X / P_{m}}^{1}(x)\right) \geqq i\right\} .
$$

The following result is known; cf. [5, Lemma 3].

Proposition. If $L$ is chosen generically, then $S_{i}$ is of pure codimension $i(m-r+i)$ in $V$, for all $i>0$.

In particular, if $m=r+1$, then $\operatorname{codim}\left(S_{1}\right)=2$, and $\operatorname{codim}\left(S_{2}\right)=6$. This says that $S_{2}=\varnothing$ if $r \leqq 5$ and $m \geqq r+1$.

Let $x$ be a closed point of $S_{1}-S_{2}$, and let $y=\pi(x)$. Let $\pi^{*}: \mathcal{O}_{p m, y} \rightarrow \mathcal{O}_{V, x}$ be the corresponding homomorphism of local rings. We can choose parameters $t_{1}, \ldots, t_{r}\left(\right.$ resp. $\left.u_{1}, \ldots, u_{m}\right)$ in $\mathcal{O}_{V, x}\left(\right.$ resp. $\left.\mathcal{O}_{p m, y}\right)$ such that $\pi^{*}\left(u_{i}\right)=t_{i}$ for $i=1, \ldots, r-1$, while $\pi^{*}\left(u_{i}\right) \in \mathrm{m}_{x}^{2}$ for $i=r, \ldots, m$, where $\mathbf{m}_{\boldsymbol{x}} \subset \mathcal{O}_{V, x}$ is the maximal ideal. In a natural way, one can define closed subschemes $S_{1}^{(q)} \subset V-S_{2}$ such that if $\operatorname{char}(k)=0$, the local generators of the sheaf of ideals defining $S_{1}^{(q)}$ are $\left(\partial^{j} u_{i} / \partial t_{r}^{j}\right)$ for $1 \leqq j \leqq q$, and $r \leqq i \leqq m$. In general, there are differential operators $D^{(j)}: \mathcal{O}_{V, x} \rightarrow \mathcal{O}_{V, x}$ such that $\left(D^{(j)} f\right)(x)$ is the coefficient of $t_{r}^{j}$ in the power series expansion of $f$ (cf. $[1, \S 16])$. The elements $D^{(j)}\left(\pi^{*} u_{i}\right)$ are the correct local generators.

AMS 1970 subject classifications. Primary 14B05, 14N05; Secondary 14M15.

Key words and phrases. Projective algebraic variety, generic projection, differential operator, Grassmann variety, Schubert cycle.

${ }^{1}$ Supported by NSF Grant GP-20550. 
THEOREM 1. Every smooth projective variety $V^{r}$ has an embedding such that if $\pi: V^{r} \rightarrow \boldsymbol{P}^{m}$ is induced by generic projection, then

(i) $S_{1}^{(q)}$ is of pure codimension $q(m-r+1)$ in $V$, for all $q$;

(ii) if $\operatorname{char}(k) \chi(q+1)($ resp. $\operatorname{char}(k) \mid(q+1))$, then $S_{1}^{(q)}$ is smooth over $k$ (resp. fails to be smooth over $k$ at only finitely many points).

The following result, which is essentially a statement about homomorphisms of formal power series rings, shows how to use Theorem 1 to obtain canonical forms for the homomorphisms $\pi^{*}: \hat{\mathcal{O}}_{p m, y} \rightarrow \hat{\mathcal{O}}_{V, x}$, when $x \notin S_{2}$.

THEOREM 2. Let $\pi: V^{r} \rightarrow W^{m}$ be a morphism of smooth varieties over $k$, with $m \geqq r$. Let $x \in V$ be a closed point, let $y=\pi(x)$, and assume that $x \in S_{1}^{(q)}-S_{1}^{(q+1)}$, and that $S_{1}^{(q)}$ is smooth at $x$. Then the local rings $\hat{\mathcal{O}}_{V, x}$ and $\hat{\mathcal{O}}_{W, y}$ can be identified with formal power series rings $k\left[\left[t_{1}, \ldots, t_{r}\right]\right]$ and $k\left[\left[u_{1}, \ldots, u_{m}\right]\right]$ so that

(i) $\pi^{*}\left(u_{i}\right)=t_{i}$, for $i=1, \ldots, r-1$;

(ii) $\pi^{*}\left(u_{r}\right)=\sum_{j=1}^{\beta} t_{q(m-r)+j} t_{r}^{j}+t_{r}^{q+1}$, where $\beta=q-1$ (resp. q) if $\operatorname{char}(k) \nmid(q+1)($ resp. $\operatorname{char}(k) \mid(q+1))$;

(iii) $\pi^{*}\left(u_{r+i}\right)=\sum_{j=1}^{q} t_{q(i-1)+j} t_{r}^{j}$, for $i=1, \ldots, m-r$.

REMARK. If $\operatorname{char}(k) \mid(q+1)$ and $q(m-r+1)=r$, then $S_{1}^{(q)}$ is finite and has no smooth points. If $\operatorname{char}(k)=2$, pinch points of surfaces in $\boldsymbol{P}^{3}$ are an example of this; we have $r=2, m=3$, and $q=1$ in this case.

The above results give information about the structure of $\hat{\mathcal{O}}_{V^{\prime}, y} / \mathfrak{p}$, where $\mathfrak{p}$ is a minimal prime ideal corresponding to a point $x \in \pi^{-1}(y)$ which satisfies the hypotheses of Theorem 2 . The next theorem can be used to show how these minimal prime ideals relate to each other.

Let $2 \leqq a \leqq m+1$, and suppose that any $a$ points of $V$ span an $(a-1)$ subspace of $\boldsymbol{P}^{n}$. Let $U_{a} \subset V \times \cdots \times V$ (a copies) consist of all $a$-tuples of distinct points. We can define a morphism $\phi: U_{a} \rightarrow G(n, a-1)$ $=\operatorname{Grass}_{a}\left(k^{n+1}\right)$, such that $\phi\left(x_{1}, \ldots, x_{a}\right)$ is the point which corresponds to the subspace $\Lambda$ spanned by $x_{1}, \ldots, x_{a}$. For a fixed $(n-m-1)$-subspace $L \subset P^{n}$, let $\Sigma \subset G(n, a-1)$ be the special Schubert cycle $\Sigma=\{\Lambda \mid$ $\operatorname{dim}(L \cap \Lambda) \geqq a-2\}$. Thus $\left(x_{1}, \ldots, x_{a}\right) \in \phi^{-1}(\Sigma)$ iff $\pi\left(x_{1}\right)=\ldots=\pi\left(x_{a}\right)$.

THEOREM 3. Every smooth projective variety has an embedding such that if $L$ is choosen generically, then $\phi^{-1}(\Sigma)$ is smooth and of codimension $(a-1) m$ in $U_{a}$. Moreover, if $a \geqq 2$, and if $q_{1}, \ldots, q_{a}$ are $\geqq 0$, then $\phi^{-1}(\Sigma) \cap\left(S_{1}^{\left(q_{1}\right)} \times \cdots \times S_{1}^{\left(q_{\mathrm{a}}\right)}\right)$ is smooth. (We set $S_{1}^{(0)}=V$.) If char $(k) \mid(q+1)$ and $S_{1}^{(q)}$ is not smooth at $x$, then $\pi^{-1}(\pi(x))=\{x\}$, provided that $m \geqq r+1$.

The fact that $\operatorname{codim}\left(\phi^{-1}(\Sigma)\right)=(a-1) m$ was proved by E. Lluis [3]; the smoothness of $\phi^{-1}(\Sigma)$ is equivalent to Theorem 1 of [4]. The proof 
of the last statement as well as the proofs of Theorems 1 and 2 will be published elsewhere.

We will now indicate how Theorem 3 is applied. Let $y \in \boldsymbol{P}^{m}$, with $\left\{x_{1}, \ldots, x_{a}\right\} \subset \pi^{-1}(y)$; assume that $x_{j} \in S_{1}^{\left(q_{j}\right)}$ for $j=1, \ldots, a$. For each $j$, we choose elements $u_{j 1}, \ldots, u_{j d_{j}}$ which form a subset of a regular system of parameters at $y$ such that each $\pi^{*}\left(u_{j v}\right)$ induces an element of the square of the maximal ideal along $S_{1}^{\left(q_{j}\right)}$ at $x_{j}$. [If $x_{j} \notin S_{1}^{\left(q_{j}+1\right)}$, and $\operatorname{char}(k) \chi\left(q_{j}+1\right)$, we can take $d_{j}=q_{j}(m-r+1)+(m-r)$.] The smoothness implies that $\left\{u_{j v} \mid 1 \leqq j \leqq a\right.$ and $\left.1 \leqq v \leqq d_{j}\right\}$ is a subset of a regular system of parameters at $y$.

In particular, let us consider the case $r=3, m=4$, with $\operatorname{char}(k) \neq 2$. In this case, there are finitely many $y \in V^{\prime}$ with $\pi^{-1}(y)=\left\{x_{1}, x_{2}\right\}$, where $x_{1} \notin S_{1}$, and $x_{2} \in S_{1}^{(1)}-S_{1}^{(2)}$. For such a point, the above considerations imply that

$$
\mathcal{O}_{V^{\prime}, y} \cong k\left[\left[u_{1}, u_{2}, u_{3}, u_{4}\right]\right] /\left(u_{1}\left(u_{2}^{2}-u_{3}^{2} u_{4}\right)\right)
$$

\section{REFERENCES}

1. A. Grothendieck, Éléments de géométrie algébrique, Inst. Hautes Études Sci. Publ. Math. No. 32 (1967). MR 39 \# 220.

2. E. Lluis Riera, Sur l'immersion des variétés algébriques, Ann. of Math. (2) 62 (1955), 120-127. MR 17, 87.

3. - On the singularities which appear in projecting algebraic varieties, Bol. Soc. Mat. Mexicana (2) 1 (1956), 1-9. MR 18, 335.

4. J. Roberts, Generic projections of algebraic varieties, Amer. J. Math. 93 (1971), 191-214. MR 43 \# 3263.

5. 1 , The variation of singular cycles in an algebraic family of morphisms, Trans. Amer. Math. Soc. 168 (1972),

Department of Mathematics, Purdue University, West Lafayette, Indiana 47907

Current address: School of Mathematics, University of Minnesota, Minneapolis, Minnesota 55455 